\title{
Prácticas de espiritualidad según Elizabeth Liebert. Aproximación a su pensamiento y ejemplificación en un estudio de caso*
}

\author{
María Marcela Mazzini** \\ Universidad Católica de Argentina \\ Argentina
}

Para citar este artículo: Mazzini, María Marcela. «Prácticas de espiritualidad según Elizabeth Liebert. Aproximación a su pensamiento y ejemplificación en un estudio de caso». Fraciscanum 169, Vol. LX (2018): 239-271.

\section{Resumen}

El objetivo del presente artículo es establecer el significado de «prácticas de espiritualidad» según Elizabeth Liebert y realizar una aplicación de esta expresión en el estudio de un caso. Luego de presentar a la autora, el concepto elegido y de señalar su importancia, se hará foco en una definición-descripción de lo que sería para Liebert una práctica de espiritualidad, desde esa perspectiva se analizarán

* Este trabajo forma parte de la producción de una beca de posdoctorado que se desarrolla en la Facultad de Teología de la UCA, sobre prácticas de espiritualidad popular en el «Hospice San Camilo», como aporte a la reflexión colectiva en el grupo de Investigación sobre «Prácticas de Espiritualidad Popular en espacios urbanos (Buenos Aires y Rosario)», perteneciente a dicha Facultad. El tema tiene también relación con lo investigado en el Seminario internacional permanente de investigación en teología de las prácticas (también llamado «grupo de Santiago»).

** Doctora en Teología por la Pontificia Universidad Católica Argentina. Docente e Investigadora en la Facultad de Teología de dicha Universidad. Miembro del grupo de Investigación sobre «Prácticas de Espiritualidad Popular en espacios urbanos (Buenos Aires y Rosario)», de la Facultad de Teología (UCA). Contacto: marcela.mazzini@gmail.com 
los puntos de contacto entre teología pastoral y la espiritualidad como disciplina académica, la relación entre práctica y experiencia y el rol que cumplen las prácticas en el desarrollo de la espiritualidad como disciplina académica. En esta primera parte se seguirán especialmente cuatro de los escritos de Liebert. En un segundo momento, se expondrá de qué modo los conceptos de Liebert sobre prácticas de espiritualidad podrían aplicarse el estudio de un caso ${ }^{1}$.

\title{
Palabras clave
}

Prácticas, espiritualidad, experiencia, pastoral, hospice.

\section{Practices of spirituality according to Elizabeth Liebert. Approximation to her thinking and exemplification in a case study}

\begin{abstract}
The objective of this article is to establish the meaning of «practices of spirituality» according to Elizabeth Liebert and to see an application of this expression in a case study. After introducing the author to the chosen concept and highlighting its importance, focus will be placed on a definition-description of what a practice of spirituality would be for Liebert and from that perspective the points of contact between pastoral theology and spirituality as academic discipline will be analyzed, as well as the relationship between practice and experience and the role of practices in the development of spirituality as an academic discipline. In this first part four of Liebert's writings will be especially followed. In a second moment, we'll try to explain how Liebert's concepts on practices of spirituality could be applied in a case study.
\end{abstract}

1 El caso del «Hospice San Camilo». 


\section{Keywords}

Practices, spirituality, experience, pastoral, hospice.

\section{Introducción}

En este trabajo se expondrá el concepto de una autora contemporánea que ha sido útil como herramienta en un estudio de caso. Se trata del concepto de prácticas de espiritualidad, la autora es Elizabeth Liebert ${ }^{2}$ y la investigación de referencia es la realizada en un Hospice (institución que acompaña los últimos días de personas de bajos recursos que tienen un pronóstico de vida de seis meses o menos) $)^{3}$.

¿Por qué elegir ese concepto en esa autora? Por los elementos que se irán detallando en el texto, el concepto de «prácticas de espiritualidad» resulta sumamente útil para comprender y exponer el tipo de espiritualidad que se vive en el «Hospice San Camilo» (HSC). Describiré dicho concepto, tal como se presenta en cuatro textos de E. Liebert. ${ }^{4}$

Elizabeth Liebert nació en 1943 en Seattle, Washington. Es religiosa de la Congregación de los Sagrados Nombres de Jesús y de María, comunidad a la que ingresó en 1962.

3 La Asociación Civil Hospice San Camilo es una ong que sustenta su accionar en la filosofía del Cuidado Hospice, la cual considera la muerte como un acontecimiento natural dentro del proceso de la vida, por ello todo enfermo merece hasta el último momento el trato conforme a su dignidad de persona. Inspirados en dicha filosofía, la Asociación prioriza a los enfermos terminales que están solos y con menos recursos, brindándoles un clima de seguridad propicio para el encuentro consigo mismos en el momento más trascendente de sus vidas. El Cuidado Hospice se ofrece en tres modalidades: en la «Casa de la Esperanza» (CDE), ubicada en Hilarión de la Quintana 2125 (1636) Olivos, Buenos Aires, Argentina, en los hospitales o en el domicilio de los huéspedes. Cf. http://www.hospicesancamilo.org.ar, consultada en diciembre 30, 2016. Cf. María Marcela Mazzini, «El Cuidado Hospice como Acompañamiento espiritual. Estudio de caso: "Hospice San Camilo"», Teología 122, Vol. LIV (2017):69-67.

4 1) Elizabeth Liebert, «The Role of Practice in the Study of Christian Spirituality», en Minding the Spirit, ed. Elizabeth Dreyer y Mark Burrows (Baltimore: John Hopkins University Press, 2005), 79-99. 2) Elizabeth Liebert, "Practice», en The Blackwell Companion in Christian Spirituality, ed. Arthur Holder (Chichester, West Sussex: Blackwell Publishing, 2005), 496-514. 3) Elizabeth Liebert, The Way of Discernment. Spiritual Practices for Decision Making (Lousville-Westminster: John Knox Press, 2008). 4) Elizabeth Liebert, The Soul of discernment. A Spiritual Practice for Communities and Institutions (Lousville-Westminster: John Knox Press, 2015). 
La autora, además de ofrecernos este interesante instrumento conceptual, es un referente en el panorama de la espiritualidad contemporánea norteamericana. La hermana Elizabeth Liebert tiene un rico itinerario espiritual y teológico que vuelca en sus cursos: completó una Maestría en Estudios Religiosos en la Universidad Gonzaga, y comenzó a enseñar en el Colegio Fort Wright en el departamento de estudios religiosos, donde también dirigió el Programa de Posgrado de Ministerios Cristianos.

Posteriormente completó un doctorado en la Universidad de Vanderbilt. Luego siguieron los años de docencia en centros de formación teológica, los últimos veintiocho en el Seminario Teológico de San Francisco y en Graduate Theological Union, en Berkeley, California, USA. Recientemente se desempeñó como Decana del Seminario y Vicepresidenta de Asuntos Académicos del San Francisco Theological Seminary, y actualmente es Profesora de Vida Espiritual y Directora del Programa de Espiritualidad Cristiana. Ella es la autora, coautora o coeditora de varios libros sobre temas de espiritualidad, focalizándose en prácticas y discernimiento ${ }^{5}$. En el mapa actual de los estudios académicos, enmarca la espiritualidad cristiana dentro de los estudios en religión, dándole así una referencia más amplia, interreligiosa e interdisciplinar al estudio académico de la espiritualidad. Enfatiza en la capacidad transformativa de la experiencia espiritual, noción sobre la cual hace foco.

En síntesis, tanto el concepto de «prácticas de espiritualidad» que ella desarrolla, como la relevancia de la autora en el panorama de la espiritualidad contemporánea, justifican ambas elecciones para la investigación realizada en el «Hospice San Camilo» que aquí se presenta.

5 Elizabeth Liebert, Changing Life Patterns: Adult Development in Spiritual Direction (New York: Paulist Press, 1992, Revised Edition, Chalice Press, 2000); Katherine Dyckman; Mary Garvin y Elizabeth Liebert, The Spiritual Exercises Reclaimed: Uncovering Liberating Possibilities for Women (New York: Paulist Press, 2001); Elizabeth Liebert y John Endres, A Retreat with the Psalms: Resources for Personal and Communal Prayer (New York: Paulist Press, 2001); Bruce Lescher y Elizabeth Liebert, Exploring Christian Spirituality: Essays in Honor of Sandra M. Schneiders (New York: Paulist Press, 2006); Elizabeth Liebert, The Way of Discernment: Spiritual Practices for Decision Making; Elizabeth Liebert, The Soul of Discernment: Spiritual Practices for Communities and Institutions. 


\section{Las prácticas de espiritualidad según E. Liebert}

Elizabeth Liebert parte de la afirmación de Miroslav Volf, según la cual, las buenas prácticas hechas en comunión constituyen la precondición para la recta comprensión de las realidades pastorales ${ }^{6}$. A partir de allí, se abren preguntas sobre el hacer, sobre las teorías acerca del hacer y sobre las prácticas concretas. En el ámbito eclesial ella dice, además, que ese estudio del hacer es diferente si el interés es la aplicación inmediata o si un académico estudia ese «hacer» de una manera teórica o general.

Propone para su reflexión un tipo particular de «hacer» al que llama «la práctica» y la analiza desde la espiritualidad como disciplina académica. Según Liebert la «práctica» es una dimensión constitutiva de la teología espiritual y útil en el diálogo con otras disciplinas teológicas ${ }^{7}$.

Para apoyar su idea la autora, en primer lugar, recurre a la comparación y al cruce espiritualidad-pastoral ${ }^{8}$. Luego de mirar algunos puntos en común y algunos contrastes, avanza con una noción que ambas disciplinas tienen en común: la experiencia. A partir de allí, saca conclusiones. A continuación, se intentará desarrollar la propuesta de la autora, siguiendo los textos que estamos analizando ${ }^{9}$.

\subsection{Teología pastoral y espiritualidad}

En la línea de reflexión sobre espiritualidad que siguen varios autores ${ }^{10}$, Liebert señala que la teología y la historia son las disciplinas que actualmente más influyen en los estudios de espiritualidad y

6 Cf. Miroslav Volf, «Theology for a Way of Life», en Practicing Theology: Beliefs and Practices in Christian Life, ed. Miroslav Volf y Dorothy Bass (Michigan-Cambridge: Eerdmans Publishing, 2002), 257. Citado en Elizabeth Liebert, «The Role of Practice in the Study of Christian Spirituality», 79.

7 Cf. Elizabeth Liebert, «Practice», 496.

8 En el presente texto, con el término «espiritualidad», salvo expresa mención, se estará nombrando la disciplina académica.

9 Ver nota 4.

10 Cf. Arthur Holder, Lisa Dahill, «Teaching Christian spirituality in seminaries today», Christian Spirituality Bulletin 7-2 (1999): 9-12. 
en los cursos que se imparten, por lo tanto no es de extrañar que estos cursos presten muy poca atención a las perspectivas teológicas bíblicas, de las ciencias sociales o de la estética ${ }^{11}$.

Liebert piensa que las vicisitudes de la teología pastoral académica ofrecen similitudes y contrastes interesantes para comprender el desarrollo de la espiritualidad entendida como disciplina científica.

Una de las razones que posibilita compararlas podría estar dada por lo siguiente: tanto la pastoral como la espiritualidad se ocupan de la «experiencia», que suele ser un concepto complejo ya sea para definir como para estudiar ${ }^{12}$. Elizabeth Liebert señala que, si bien un énfasis sobre la experiencia es importante tanto para el estudio de la teología pastoral como para la espiritualidad, el término «práctica» (central en la teología pastoral), es particularmente útil en la interrelación entre la espiritualidad vivida y el estudio académico de la espiritualidad ${ }^{13}$.

Liebert entiende que, en su sentido más amplio, la teología pastoral trata de relacionar los significados y las exigencias de la fe con los problemas humanos y las situaciones concretas, utilizando la experiencia humana para llegar a una comprensión más profunda de Dios. En este empeño, frente a cada coyuntura, ya sea un hecho, un vínculo, una liturgia, una crisis de la vida, se pregunta: ¿Qué significa? ¿Qué se pide de mí, de los demás? ¿Cómo afecta esto que sucede, la comprensión que tenemos de Dios y la relación que establecemos con él?

11 Cf. Elizabeth Liebert, «Practice», 497.

12 Hay distintas definiciones de experiencia con diferentes puntos de vista. Por ejemplo: Hans Urs von Balthasar, Gloria. Una estética teológica (Madrid: Encuentro, 1985), Vol 1. 203ss; Tullo Goffi, La experiencia espiritual, hoy. Líneas esenciales de la espiritualidad cristiana contemporánea (Salamanca: Sígueme, 1987), 115-163; Rosa Cursach Salas, «Experiencia. La experiencia de las mujeres en la teología feminista. Prácticas de teología fundamental feminista», en 10 palabras clave en Teología Feminista, ed. Mercedes Navarro y Pilar de Miguel (Navarra: Verbo Divino, 2004), $17-$ 55; Pedro Rodríguez Panizo, «Experiencia religiosa», en Nuevo Diccionario de Teología, dir. Juan José Tamayo (Madrid: Trotta, 2005), 358-367

13 La misma idea se encuentra en Claire Wolfteich, «Animating Questions: Spirituality and Practical Theology", IJPT 13, (2009): 121-143 
Del mismo modo, las preguntas recorren el camino opuesto: $¿ \mathrm{De}$ qué manera mi comprensión y relación con Dios afecta a la interpretación de esta experiencia particular? Si mi manera de comprender a Dios, a Cristo, a la Iglesia, son de un determinado modo, entonces, ¿qué significa este terremoto, por ejemplo?

Para Liebert, la naturaleza de la teología pastoral es dinámica, pudiéndose definir como la tarea de reflexión que, desde la oración y el compromiso cristiano, considera todos los aspectos de un evento o caso particular: la experiencia del individuo, la experiencia del ministro, la experiencia de la comunidad, el aspecto sociológico, cultural, psicológico, etc. Todos estos aspectos se ponen en diálogo con la tradición cristiana en toda su riqueza y pluralidad, es decir, con las Sagradas Escrituras y con los documentos fundacionales de la comunidad de fe.

De la consideración integral de estos aspectos que dialogan entre sí, surgen respuestas teológico-pastorales en tres direcciones: hacia la situación en sí misma, hacia la práctica pastoral en particular y hacia la reflexión teológica en general. Esta descripción se centra en el momento del «hacer» de la teología pastoral y deja suponer el paso previo de una cuidadosa descripción del caso $^{14}$.

Al centrarse en el proceso más que el contenido, la descripción que Liebert propone se parece bastante al círculo hermenéutico de la teología de la liberación, que ilumina la naturaleza dinámica y repetitiva de un proceso para determinar la acción teniendo en cuenta su contexto ${ }^{15}$. Así el círculo hermenéutico que la autora propone va de la experiencia, al análisis, del análisis a la oración y reflexión teológica y de la oración y reflexión, a la acción pastoral. Este círculo

14 Es interesante como describe «el caso» - «el estudio de casos» en la versión del artículo Elizabeth Liebert, «The Role of Practice in the Study of Christian Spirituality», 79-99. En la nota 7 del mencionado artículo dice que por «caso» ella entiende una situación, hecho, dilema o sistema, que ha sido lo suficientemente abstraído de la coyuntura, como para poder ser descripto. También se refiere a lo que se llama «descripción densa» de una situación.

15 Este círculo y su descripción, aparecen a menudo en los escritos de la autora, incluso en su último libro: Elizabeth Liebert, The Soul of discernment. A Spiritual Practice for Communities and Institutions. En la Introducción, posición 204 de la versión Kindle. 
puede repetirse indefinidamente y describe más un proceso que una realidad estática.

En opinión de Liebert, los teólogos sistemáticos a menudo parecen no tomar en serio a los teólogos pastorales. Esto es así, porque los sistemáticos piensan que los teólogos pastorales tratan sus temas con métodos blandos, involucrándose en el pensamiento teológico de modo descuidado y confuso, tratando sobre todo con particularidades de la experiencia humana. Estas mismas sospechas recaen sobre la disciplina académica de la espiritualidad ${ }^{16}$.

Hoy, en el campo de la teología pastoral, al igual que en el estudio académico de la espiritualidad, hay una pluralidad de interlocutores. Grupos de distintas disciplinas académicas les preguntan a la teología pastoral y a la espiritualidad: «¿Cuáles son los límites de la disciplina?» Ambas intentan centrar su ámbito de dos maneras: por la elaboración de una definición que las delimite y seleccionando los métodos apropiados a sus investigaciones.

En cuanto a los parámetros metodológicos, los teólogos pastorales son guiados por un caso particular, o una situación, y esta determina la gama de metodologías adecuadas para la integración de la teoría y la práctica en una situación dada. Análogamente, en el estudio académico de la espiritualidad, la pregunta de investigación determina los interlocutores y la metodología, que se centrarán en la experiencia de lo sagrado.

Para los teólogos pastorales, como para los estudiosos de la espiritualidad cristiana, el caso particular es en sí importante y digno de reflexión crítica. Se trata de la «materia» que estudia la disciplina. Estas «disciplinas problemáticas», llamadas así porque entran en juego con la problemática del fenómeno a ser estudiado como, por ejemplo: psicología, sociología, literatura, etc. ${ }^{17}$, son precisamente

16 Elizabeth Liebert, «The Role of Practice in the Study of Christian Spirituality», 84.

17 La expresión «disciplinas problemáticas» es de Sandra Schneiders. Dicha autora afirma también que el término «espiritualidad», es inevitablemente ambiguo, puede hacer referencia tanto a una dimensión fundamental del ser humano, la experiencia vivida en esa dimensión y la disciplina 
aquellas que entenderán mejor que ninguna otra la experiencia (el caso) en orden a buscar un mayor conocimiento, precisión y empatía, (y lo más importante a juicio de Liebert), para ayudar a las personas o grupos a entender su propia experiencia y la de otros en mayor profundidad. En todos estos aspectos la teología pastoral y la espiritualidad están muy próximas.

Tanto la «teología pastoral», como la «espiritualidad cristiana», pueden referirse en diferentes momentos al fenómeno (caso), a la reflexión en profundidad sobre dicho caso (el hacer concreto de la teología pastoral o de la espiritualidad), y al estudio académico de la historia, los métodos y el aporte de las otras disciplinas.

La diferencia más importante que Liebert encuentra entre ambas es la siguiente: mientras la pastoral, pone el foco directamente en la praxis, preocupada por acompañar distintas situaciones en las comunidades, la espiritualidad se concentra en la experiencia de lo sagrado, tanto cuando dicha dimensión se manifiesta en lo personal como en lo comunitario. El gran punto de encuentro entre ambas disciplinas es la experiencia ${ }^{18}$. A continuación, se desarrollará parte de esta cuestión.

\subsection{La experiencia}

La teología pastoral revela su predilección por la reflexión crítica sobre la experiencia en su profundización en el evento o situación en particular: el caso. La espiritualidad cristiana se dedica a reflexionar sobre la experiencia religiosa cristiana como tal, en la naturaleza auto-implicativa de dicho estudio. Muchos autores, entre ellos y ellas, Sandra Schneiders ${ }^{19}$, insisten en señalar que el estudio de

académica que estudia esa experiencia. Cf. Sandra Schneiders, "Spirituality in the Academy», Theological Studies 50, (1989): 676-697. En esa misma línea: Kees Waaijman, «Toward a phenomenological definition of Spirituality», Studies in Spirituality 3 (1993): 5-56. Ver también el contexto más amplio de estatuto epistemológico de la espiritualidad tal como lo expone Azcuy en: Virginia Azcuy, «La Espiritualidad como Disciplina Teológica. Panorama Histórico, Consensos y Perspectivas actuales», Teología 105 (2011): 251-280.

18 Cf. Elizabeth Liebert, «Practice», 500.

19 Cf. Sandra Schneiders, «Spirituality in the Academy», 676-697. 
la espiritualidad cristiana, como una disciplina académica, debe estar conectado con la realidad, en particular con la realidad que se estudia. No basta estudiar espiritualidad en los libros, ese estudio debe correr parejo con una cierta experiencia del evento estudiado ${ }^{20}$. Claramente, Liebert se alinea aquí con Schneiders.

Pero, iqué es exactamente lo que queremos decir con «la experiencia»? Muchas veces se asume que el significado es evidente por sí mismo, pero en la práctica es un tema complejo. Liebert prefiere seguir las categorías de la teología pastoral para desentrañar el significado de este término escurridizo.

La experiencia, dice el teólogo pastoral Brian Childs, es «la participación o el encuentro con la realidad $»^{21}$. El término también puede referirse al conocimiento práctico adquirido a través de dicha participación o encuentro con la realidad, es lo que nos ha sucedido, lo que hemos hecho con aquello que nos sucedió y las formas en que hemos aprendido algo de lo que hicimos y vivimos.

Si contraponemos experiencia y reflexión, una habitual y desafortunada dicotomía que haríamos bien en evitar, la experiencia destaca la inmediatez en contraposición a la reflexión sobre esa experiencia, reflexión que establece una cierta mediación con los hechos. La experiencia en realidad incluye la reflexión, así como la inmediatez inicial.

Es inherente a la experiencia este sentido integral, holístico, englobante. Hacer una experiencia o tener una experiencia es, de algún modo, verse envuelto en una realidad que nos toma y de la que participamos. Además, la experiencia humana se desarrolla dentro de una red de relaciones. Estas relaciones proporcionan el contenido, los objetos, el ambiente y el contexto de la vivencia. La experiencia proporciona el material a partir del cual se forman los recuerdos,

20 Cf. Mary Frohlich, «Spiritual Discipline, Discipline of Spirituality: Revisiting Questions of Definition and Method», Spiritus 1, Vol. 1 (2001): 65-78.

21 Brian Childs, «Experience», en Dictionary of Pastoral Care and Counseling, ed. Rodney J. Hunter (Nashville: Abingdon Press, 1990), 388. 
pensamientos, imágenes, sentimientos y decisiones. La experiencia comprende tanto el mundo sobre el que actuamos, como los «otros» con quienes se establecen relaciones diversas: la naturaleza, uno mismo, los demás seres humanos, las estructuras socio-políticas, y lo trascendente.

Se suele asociar la experiencia con una opinión personal, efímera, superficial. Es muy iluminadora la reflexión de Bellet:

\begin{abstract}
Algunas personas -especialmente los teólogos- pueden fruncir el ceño, diciendo que la experiencia es algo puramente subjetivo. Pero, żqué es esa distribución entre subjetivo y objetivo? Como si la experiencia estuviese del lado de lo subjetivo y la doctrina del lado de lo objetivo. Estas son categorías mortales. Si la doctrina está del lado de lo objetivo que excluye la experiencia, la doctrina se transforma en algo doctrinario. Si la experiencia está del lado de lo subjetivo, significa que no puede haber experiencia de aquello que afirma la doctrina ${ }^{22}$.
\end{abstract}

Tanto Liebert como Bellet (aunque en otro contexto), revalorizan la experiencia como punto de encuentro con lo real. No existe «un saber» separado de la experiencia personal y comunitaria. A todos los contenidos es posible acceder desde un lugar existencial, ese es el lugar de la experiencia, lugar por cierto limitado y condicionado, que amerita ponerse en diálogo con otros lugares. Además, la experiencia aunque sea personal y solitaria se da siempre en el contexto de una comunidad de referencia. Fuera de ese contexto no es posible pensar la realidad.

\title{
1.3 La "práctica» en el estudio académico de la espiritualidad
}

Liebert constata que existe un debate en la academia, sobre la cuestión de la admisibilidad de la "práctica» como parte del objeto de estudio de la espiritualidad.

Dos preguntas básicas en este sentido serían: ¿Qué es la «práctica»? ¿Cómo se relaciona con la experiencia? En términos generales, Liebert adhiere a la definición de Rebecca Chopp ${ }^{23}$ : las 
prácticas son formas de comportamiento social que median entre lo subjetivo y lo objetivo. Una práctica es un patrón de significado y acción que está culturalmente construido y que tiene una instancia individual. La noción de la práctica nos lleva a indagar en las actividades compartidas en los grupos de personas, dichas prácticas proporcionan sentido y orientación al mundo de los que adhieren a ellas y guían su acción.

En términos de espiritualidad cristiana, Liebert propone utilizar el término "práctica» entendiéndola como la acción intencional y repetida de la espiritualidad vivida en los diversos ámbitos. Dichas prácticas pueden ser objeto de estudio académico y ese estudio tiene por objetivo atender y entender lo que ocurre cuando estas se desarrollan ${ }^{24}$. Deliberadamente elige la palabra «práctica» porque en inglés existe como sustantivo, pero también como verbo, es como si en español nombráramos con la misma palabra el sustantivo "práctica» y el verbo «practicar», el término adquiere así, una dimensión muy fuerte. Normalmente connota una disciplina espiritual particular, pero tal como la usa Liebert, "práctica» se refiere a la actividad de la espiritualidad, que continuamente está trayendo «practicantes» a la academia, que estudiarán sus propias experiencias compartidas por una comunidad ${ }^{25}$.

Hablar de o estudiar una «espiritualidad vivida», es asistir con la mayor autenticidad posible a la verdad de la propia experiencia, incluida la verdad del otro que resulta desafiante y descentralizadora.

Liebert trata de provocar intencionalmente la experiencia en sus cursos, porque entiende que la espiritualidad vivida, sigue siendo el foco de compromiso para cualquier estudio de la espiritualidad. Por lo tanto, los estudiosos de las prácticas de la espiritualidad cristiana habrán de reflexionar juntos sobre la verdad de la experiencia hasta que este modo de trabajo se convierta en una "segunda»

24 Cf. Elizabeth Liebert, «Practice», 503.

25 Es notable como Liebert insiste en el aspecto autoimplicativo y comunitario del estudio de la espiritualidad. 
naturaleza y permita valorar el sentido y el alcance de las prácticas de espiritualidad de otras personas y grupos ${ }^{26}$.

Así Liebert amplía el estudio académico de la espiritualidad, que sería mucho más que llevar las disciplinas espirituales clásicas o contemporáneas a las aulas, aunque eso también podría incluirse en determinadas circunstancias y de hecho se incluye. Es cuestión de llevar a la práctica lo que se estudia y de estudiar y reflexionar críticamente lo que se hace. Una «práctica de espiritualidad» es la experiencia compartida y la auto-reflexión crítica de la espiritualidad vivida.

La misma autora se imagina el tipo de objeciones a estas afirmaciones que surgen inevitablemente: «iCómo se mantendrá el rigor académico necesario?» "Eso podría darse en un aula de seminario, pero no es posible en la universidad». «Eso puede funcionar con algunos temas contemporáneos o más existenciales, pero no va a funcionar para los textos antiguos o clásicos», etc.

Sandra Schneiders ha señalado con gran lucidez que uno de los principales desafíos con los que la disciplina de la espiritualidad se enfrenta a medida que se desarrolla su identidad en la academia, es el siguiente: ¿Cómo integrar un enfoque holístico a la investigación con plena rendición de cuentas a los estándares de la ciencia teológica?, o en otros términos: ¿̇cómo desarrollar un compromiso personal con lo que se está estudiando, a la vez que se lleva adelante una perspectiva metodológica adecuada? ?7 $^{27}$

Liebert ofrece un ejemplo de su propia tarea docente: da un curso de lectio divina para estudiantes de teología que tiene como objetivo enseñar una forma de oración que encuentren favorable en su propia vida personal y espiritual. Se propone mostrar un

26 Este tema está planteado de un modo creativo, al hablar de las prácticas para el discernimiento grupal en Elizabeth Liebert, The Way of Discernment. Spiritual Practices for Decision Making.

27 Cf. Sandra Schneiders, "A Hermeneutical Approach to the Study of Christian Spirituality», en Minding the Spirit, ed. Elizabeth Dreyer y Mark Burrows (Baltimore: John Hopkins University Press, 2005), 49-60. 
modo de acceso para mejorar y profundizar en los textos bíblicos en orden a la preparación de la predicación, pensando especialmente en los pastores o futuros pastores, y ofrecer un método de oración comunitaria útil en muchos entornos eclesiales ${ }^{28}$.

Ella acepta que tiene como una segunda intención en todo lo que enseña y que ese propósito es francamente pedagógico: involucrar al estudiante en una variedad de vías para apropiarse del material según lo sugerido por el propio material. Yendo al ejemplo de la lectio divina, una cosa es solamente estudiar qué es y cuáles son sus pasos según diversos autores y escuelas de espiritualidad. Otra perspectiva, (que es la que Liebert desarrolla), consiste en hacer ese estudio pero además proponerles a los alumnos que ellos hagan lectio divina y que luego reflexionen sobre su experiencia, estableciendo conexiones con lo previamente estudiado.

Quiere ofrecer muchas avenidas de acceso para que los estudiantes realmente puedan entender y ser captados por el material que estén investigando juntos. Es decir, pretende crear un espacio donde la naturaleza auto-implicativa y transformadora de la disciplina potencialmente pueda echar raíces. Cuando la experiencia «entra en el aula», el estudio de dicha experiencia se vuelve inmediato y convincente. La experiencia forma parte del aprendizaje no tanto cuando se incorpora a través de los ejemplos o la casuística, sino cuando se enseña utilizando la experiencia misma de los sujetos implicados en ese aprendizaje. Es importante tener en cuenta que, tanto la espiritualidad, como la religión, tratan, precisamente, sobre

Aquí se hace referencia a las diversas iglesias cristianas de donde vienen sus alumnos. Elizabeth Liebert es religiosa católica, pero su institución de referencia es «San Francisco Theological Seminary», donde ella es profesora y directora del programa de Espiritualidad Cristiana. Dicha institución es de la Iglesia Presbiteriana y recibe alumnos de distintas denominaciones cristianas. SFTS es miembro de Graduate Theological Union (GTU) y tiene convenio con distintas universidades de la costa oeste de usa. Por ello tiene de base una perspectiva intercultural, ecuménica e interdisciplinar. De hecho GTU ha cambiado nuevamente en 2016 su plan de estudios de doctorado, reforzando el acento en la experiencia, en le interculturalidad y en la interdisciplina. consultada en diciembre 22, 2016 http://gtu.edu/academics/doctoral-program 
la forma de generar orden y significado en la propia vida en relación con uno mismo, con los demás y con Dios ${ }^{29}$.

En la posmodernidad, se admite que no hay puntos de partida neutros o puntos permanentes. Lo que cada persona cree y ha experimentado, inevitablemente influye en lo que sabe y en cómo lo enseña, llegado el caso.

Liebert afirma que la reflexión sistemática sobre las prácticas, esto es, sobre la espiritualidad vivida es constitutiva de la disciplina de la espiritualidad cristiana ${ }^{30}$.

Se ha de enseñar reflexionando sobre las prácticas, «ejercer nuestra práctica», por así decirlo, con el fin de que se convierta en una perspectiva metodológica intencional por los materiales con los que nos involucramos, siendo conscientes que, al transmitir la experiencia, transmitimos una idea del sentido de la vida.

\subsection{Conclusión: la importancia de la práctica en la espiritualidad como disciplina académica}

La práctica ofrece un posible marco para el acceso a la experiencia inmediata de la vida espiritual, objeto de la disciplina. Cuando la experiencia «entra en el aula», como se ha dicho, el estudio de la experiencia de la vida cristiana se vuelve inmediato y convincente.

Muchos estudiosos de la espiritualidad cristiana trabajan casi exclusivamente con textos: bíblicos o de diferentes géneros literarios que han sido reconocidos como parte de nuestra herencia cristiana, o dejados por importantes pensadores, teólogos, pastores y maestros espirituales. La pregunta clave que nuestra disciplina plantea a los textos de todas clases y épocas sería: ¿̇Cuál es la experiencia espiritual vivida que el texto refleja/transmite? ¿Cuál es la experiencia que le

Esto lo señalan muy bien los siguientes autores: Ruard Ganzevoort - Johan Roeland, «Lived religion: the praxis of Practical Theology», IJPT 18 Vol. 1 (2014): 91-101. Los autores invitan a prestarle atención a prácticas que no son tradicionalmente religiosas, pero que ayudan a las personas a conectarse con lo sagrado y con el sentido de la vida y de la muerte. Esas prácticas, son accesos a nuevas formas de espiritualidad y religión.

30 Cf. Elizabeth Liebert, «Practice», 507. 
dio origen? ¿Cuál es la experiencia que tuvieron los primeros oyentes o lectores del texto, (en la medida en que se pueda saber)?, Liebert se pregunta: ¿Qué experiencia suscita en mí y en mis estudiantes hoy este texto? A la luz de esta discusión, ahora se puede pedir más: ¿Qué experiencia nueva se crea en el acto de la investigación de este texto, teniendo en cuenta su procedencia, su interpretación, y su utilidad existencial? ¿Qué experiencias de mi propia vida me ayudarían a entrar fielmente en el mundo de este texto? ¿Qué prácticas compartidas nos facilitarían el hecho de entender este texto y su mundo y a nosotros mismos como intérpretes? ${ }^{31}$.

Esto no quiere decir que un individuo puede simplemente leerse a sí mismo en el texto, o manipularlo artificialmente para que el texto diga algo que quiere leer allí. Deben darse todos los pasos exegéticos apropiados, incluyendo el establecimiento de la exactitud del texto, la adecuación de la traducción empleada, el estudio serio del contexto, el autor, la comunidad de origen, la forma literaria, la intención del autor, etc.

También debemos tratar de ser conscientes de nuestras propias suposiciones y prejuicios y tratar de dejarlos de lado durante la fase de investigación de nuestro trabajo con el texto. Pero cuando ese trabajo de base se ha hecho, ¿̇se permite que la práctica de la espiritualidad vivida nos ayude a repensar el texto? Liebert tiene la impresión, compartida por la presente investigación, que ese trabajo completo e integrador, no se hace habitualmente.

El cuidadoso trabajo del erudito, puede ser a la vez autoimplicativo y transformador, no solo en nuestros puntos de vista teóricos precisamente, sino también en la forma en que rezamos, actuamos y vivimos. Esta apropiación holística está en gran medida aún pendiente.

31 Cf. Elizabeth Liebert, «Practice», 509-510. 
¿Qué podría aportar esta noción de la práctica como acción intencional y repetida de la espiritualidad vivida, al trabajo académico en esta disciplina?

La «práctica» es constitutiva de la espiritualidad como disciplina académica. ¿ Puede haber demasiada práctica en el aula? La pregunta surge naturalmente cuando se utilizan prácticas como medio de atraer a los estudiantes hacia el tema en el que el profesor desea profundizar o hacer foco. Pero cuando la práctica se desplaza hacia la comprensión, entendida como la reflexión crítica de la espiritualidad vivida, entonces se transforma inmediatamente en aprendizaje. En este punto, vemos el profundo sentido que tiene traer las prácticas al trabajo académico en espiritualidad.

Liebert se alinea así con el trabajo de Schneiders, cuando esta última propone para la espiritualidad como disciplina académica, una hermenéutica en tres tiempos, al estilo de Paul Ricoeur: primera comprensión (encuentro con el tema), profundización (análisis de las fuentes, investigación y búsqueda de nuevos datos) y comprensión enriquecida o nueva compresión. En el tercer momento correspondería poner en diálogo lo investigado, con las propias prácticas, allí lo auto-implicativo tiene su lugar ${ }^{32}$.

La expresión «práctica de espiritualidad» es una expresión feliz en cuanto manifiesta precisamente un trozo de la espiritualidad vivida tal como Elizabeth Liebert describe. Hablar de prácticas de espiritualidad, remite a la observación de concreciones de la espiritualidad y al mismo tiempo, a la posibilidad de estudiar esas expresiones en los tres tiempos de la hermenéutica antes detallada. Ese estudio y su hermenéutica requieren explorar nuevos caminos y utilizar herramientas investigativas tal vez poco utilizadas hasta el momento ${ }^{33}$. Estos caminos nuevos tienen su complejidad, pero

32 Cf. Bruce Lescher y Elizabeth Liebert, Exploring Christian Spirituality: Essays in Honor of Sandra Schneiders, 3-5.

33 En nuestro grupo se sigue el método cualitativo de las ciencias sociales y se realizan entrevistas a profundidad. 
también una gran riqueza, siendo uno de sus principales méritos el contacto directo con la realidad, hecho que suscita un gran interés tanto en los estudiosos como en los que realizan las prácticas (los «practicantes») por el hecho de que ese estudio les permite analizar, corregir, enriquecer y mejorar sus acciones. De este modo la teología académica revela una faceta muy interesante de servicio hacia la praxis pastoral.

\section{Un intento de ejemplificación de un estudio de caso: las prácticas de espiritualidad en el «Hospice San Camilo»}

A continuación, haremos foco en algunas ideas Liebert poniéndolas en relación con la investigación que se lleva adelante en el «HSC». Como ya se expuso en la introducción a este trabajo, relevar las prácticas de espiritualidad que se dan el Hospice, ha resultado un método útil para comprender la particular experiencia que allí tienen los huéspedes y todas las personas implicadas en el cuidado de los mismos.

\subsection{La «espiritualidad vivida» en el ámbito de los cuidados Hospice}

En los cuidados paliativos/hospice, se maneja un concepto de espiritualidad que la define como aquello que vincula a la persona con un anhelo de profundidad y plenitud, que se realiza a través de alguna manera de trascendencia y que tiene mucho que ver con el abanico relacional:

«La espiritualidad es un universal humano, expresión de ese dinamismo que impulsa nuestro anhelo inagotable de plenitud y felicidad que caracteriza la condición humana y que se expresa en la red de relaciones que cada persona establece.

Esta naturaleza espiritual, profunda, íntima e intangible que nos caracteriza y confiere nuestra humanidad, es dinamismo que pertenece y se experimenta a un nivel transracional, transpersonal y transconfesional. 
Es decir se descubre y se vive en, pero sobre todo más allá de lo cognitivo, de lo individual y de lo creencial, trascendiéndolo e integrándolo» ${ }^{34}$.

Sobre todo, en cuidados paliativos/hospice, se insiste en que la espiritualidad es una dimensión del ser humano que se encuentra más allá o más acá de las religiones. En esta conceptualización la espiritualidad no se identifica con la religión, aunque en algunas personas su dimensión espiritual se exprese a través de alguna religión determinada.

«En los últimos años se está dando un fenómeno cada vez más extendido: considerar que la espiritualidad es una dimensión esencial del ser humano sin la cual quedamos seriamente amputados. Ello no supone necesariamente un retorno a las religiones, sino que se trata de algo nuevo que no se había dado hasta ahora: distinguir la espiritualidad de las religiones tal como actualmente las conocemos» ${ }^{35}$.

En la proximidad de la muerte, en general esta dimensión espiritual se hace más tangible o evidente. La conciencia de morir invita a asomarse a aspectos de la vida no explorados habitualmente y en especial, a este nivel de la existencia. Quienes tienen incorporada la dimensión espiritual, la intensifican y quienes no la han frecuentado antes, en general al menos la exploran. El sufrimiento, que suele acompañar la proximidad de la muerte, tal como indica también Melloni en el referido artículo ${ }^{36}$, también promueve un ejercicio de interioridad que suele ir acompañado de un desvelamiento del sentido de la vida, de la historia personal, vincular, etc.

34 Enric Benito, Javier Barbero, Mónica Dones y Julio Gómez, «Modelo de acompañamiento espiritual. Propuesta del grupo de trabajo de espiritualidad SECPAL», en Espiritualidad en Clínica. Una propuesta de Evaluación y Acompañamiento Espiritual en Cuidados Paliativos, ed. Enric Benito, Javier Barbero y Mónica Dones (Buenos Aires: Ed Biblos-Instituto Pallium Latinoamérica, 2015), 271.

35 Javier Melloni, «La Espiritualidad como Universal Humano», en Espiritualidad en Clínica. Una propuesta de Evaluación y Acompañamiento Espiritual en Cuidados Paliativos ed. Enric Benito, Javier Barbero y Mónica Dones (Buenos Aires: Ed Biblos-Instituto Pallium Latinoamérica, 2015), 39. Ver también el consenso o acuerdo de Passadena, en el que se formulan algunas claves para el acompañamiento espiritual en cuidados paliativos. Cf. Christina Puchalski, «La mejora de la calidad de los cuidados espirituales como una dimensión de los cuidados paliativos: el informe de la Conferencia de Consenso", Medicina Paliativa 18 Vol.1 (2011): 55-78.

36 Cf. Javier Melloni, «La Espiritualidad como Universal Humano», en Espiritualidad en Clínica. Una propuesta de Evaluación y Acompañamiento Espiritual en Cuidados Paliativos, 39-43. 
Es un tema largo para desarrollar aquí, pero se pueden señalar al respecto, dos elementos que aportan al punto que nos interesa marcar en este lugar. El primero: la espiritualidad como dimensión humana cobra especial relevancia y significatividad en el final de la vida y, en segundo lugar, es importante acompañar a las personas y prestar atención a las prácticas que vehiculizan la experiencia espiritual en este tramo crucial de la biografía.

Comprender las necesidades espirituales de seres humanos que están en el final de la vida, nos va a llevar a prestar atención a esas formas de comportamiento social que son las prácticas, como mediaciones entre lo subjetivo y lo objetivo, como patrones de significado y acción. Las prácticas nos dirán qué es importante y qué no lo es para cada huésped.

A su vez el estudio de esas prácticas puede arrojar mucha luz a nuestra comprensión teórica y empírica de la espiritualidad en el final de la vida. Traer al aula con caridad fraterna y con rigor académico las prácticas espirituales de los enfermos terminales, es una tarea audaz y movilizante, pero plena de sentido y que produce aprendizajes válidos y duraderos ${ }^{37}$.

\subsection{La experiencia como lugar de cruce entre la espiritualidad y la praxis pastoral}

En las entrevistas y en la observación participante que hacemos en el Hospice, aparece claramente tanto la centralidad de la experiencia, como la dificultad y a la vez la necesidad de definirla. Vemos también que la experiencia espiritual de huéspedes, enfermeras, voluntarios y todos aquellos que participan de ese espacio, tiende a plasmarse en algunas acciones: participación en la celebración de la Palabra

37 De hecho, así sucedió cuando se expuso en el curso de Teología Espiritual II (en 2015 y en 2016), en la Facultad de Teología de la UCA, algunas de las prácticas encontradas durante la investigación en el «Hospice San Camilo». La asignatura toma el tema del acompañamiento espiritual y a través de las prácticas del Hospice, se pudo abordar la cuestión del acompañamiento en el final de la vida y el acompañamiento en los procesos de duelo. En las evaluaciones que hicieron los alumnos del curso, señalaron que ese tema era uno sobre los que más habían aprendido de la materia a nivel teórico y sobre el que habían sacado mayor provecho espiritual y pastoral. 
que se hace diariamente en el Hospice, charlas con los voluntarios/ as, con la gente de la pastoral de la escucha ${ }^{38}$, celebración de la luz, en general todo lo que englobamos en el «cuidado compasivo» ${ }^{39}$.

Siguiendo la definición de Liebert, podríamos decir que son todas prácticas de espiritualidad. En el caso del Hospice se ve que las prácticas son «espiritualidad vivida», actos que median entre lo subjetivo y lo objetivo, que son profundamente personales, pero a la vez compartidos y que tienden a resignificar experiencias y en muchos casos contribuyen a sanar situaciones dolorosas de la historia personal.

Las experiencias, cuando son relatadas y reflexionadas, permiten afinar las prácticas. Por ejemplo: uno de los huéspedes relató cuánto significaba para él la celebración diaria de la Palabra. Aunque él no podía levantarse de la cama, escuchaba que en el hall se desarrollaba la celebración y eso le daba una sensación de compañía, de paz, de esperanza ${ }^{40}$. Uno de los voluntarios, que se interesa por el tema de la celebración, tomó conocimiento de esta experiencia del huésped y consultó a los otros huéspedes que estaban en ese momento en la "Casa de la Esperanza», recibiendo testimonios similares. A partir de esos testimonios y luego de conversar el tema con las coordinadoras y con los otros voluntarios, se decidió que cuando fuera posible cantarían y/o tocarían la guitarra en la celebración para que los huéspedes que no se pueden movilizar escuchen y participen más de la misma ${ }^{41}$.

Este es un ejemplo pequeño pero claro, de un hecho que luego de ser percibido, analizado y discernido en comunidad, impulsa a tomar una acción que redunda en una mejora de una práctica concreta.

38 Se trata de un grupo que va al Hospice, con el objetivo de escuchar tanto a los huéspedes como a los voluntarios, enfermeras, etc. Es una práctica pastoral y espiritual que se está desarrollando en la diócesis de San Isidro, Buenos Aires, Argentina (la diócesis en la que se encuentra el Hospice) y que se da en distintos grupos: pastoral de juventud, pastoral de la salud, tercera edad, etc.

39 Cf. María Marcela Mazzini, «El Cuidado Hospice como Acompañamiento espiritual. Estudio de caso: "Hospice San Camilo"», Teología 122, Vol. LIV (2017): 69-97.

40 Testimonio recogido el 2 de diciembre de 2015 en la Casa de la Esperanza.

41 Se ofrece la posibilidad de recibir la Eucaristía, a quienes lo deseen. Respecto del ejemplo, es interesante cómo (en general) a los huéspedes les gusta y les hace bien la música. No es raro que pidan en sus momentos finales que alguien cante o toque la guitarra y que se ejecuten canciones religiosas. 


\subsection{La cuestión del sentido en el «Hospice San Camilo»}

En este contexto es muy importante la noción de sentido. Percibir que la propia biografía tiene un sentido o que es posible dárselo, aunque la persona se encuentre al final de la vida, es una de las tareas centrales a realizar en cuidados paliativos ${ }^{42}$.

Las prácticas están muy conectadas con el sentido, con el sentido de la vida y de la historia en general y con el sentido de la propia historia en particular. A través de su práctica de espiritualidad la persona o el grupo:

- buscan conectar con el sentido, que ya se conoce o se sabe. Por ejemplo, cuando la noción de sentido está relacionada con lo cristiano, y alguien intenta unir el propio dolor a la pasión de Jesús, buscando cómo hacerlo. En este caso hay una búsqueda de establecer la relación entre algo de lo vivido y una realidad plena de significado para la persona, entonces los hechos puntuales empiezan a participar de un sentido mayor y evidente para el sujeto.

- o bien encontrar un sentido que se presiente o se cree pero que no se encuentra. Por ejemplo, cuando la persona intuye que lo que está viviendo debe tener alguna razón, pero la desconoce, reflexiona y se da cuenta de la razón de ser de un hecho o de un período de la vida.

- o bien darle un sentido a algo. En algunas ocasiones, las personas se dan cuenta, sienten o perciben que lo que sucede no está en sus manos, pero que pueden «hacer algo» con esa experiencia, que está en su libertad el hecho de que sea una experiencia alienante o liberadora.

Esto es muy claro en el HSC, con las prácticas como condensación de espiritualidad vivida, se busca expresar el sentido, transitarlo,

42 Sigo en este tema como marco general a Enric Benito, Javier Barbero, Mónica Dones y Julio Gómez, «Modelo de acompañamiento espiritual. Propuesta del grupo de trabajo de espiritualidad SECPAL», en Espiritualidad en Clínica. Una propuesta de Evaluación y Acompañamiento Espiritual en Cuidados Paliativos, 271-279. 
darle sentido a la muerte y al morir. Dicho sentido puede aparecer como conexión, como un ponerse en una línea de coherencia con el Todo, o bien como la percepción de una «revelación», un darse cuenta de por qué sucede lo que sucede o bien como una decisión, como una orientación libre que se le da a lo no elegido, como la muerte o la enfermedad.

Hay una experiencia que se repite bastante: la conciencia de aprovechar el tiempo valioso del final ${ }^{43}$, como un tiempo propicio, un kairós para revisar la propia vida y cerrar círculos abiertos (cada uno lo hace como puede). El amor que se ofrece en la «Casa de la esperanza» es el ámbito propicio que permite que las personas puedan realizar estas tareas. Al calor de la contención que ofrecen los/as voluntarios/ as, el capellán, las enfermeras, los huéspedes se animan a revisar sus asuntos pendientes y darles trámite. A veces estas charlas se dan con la gente del ministerio de la escucha, pero lo que he observado en las visitas al Hospice (y además lo han referido la Dra. Isabel Pincemín ${ }^{44}$ y la Lic. Cynthia Alvarado ${ }^{45}$ ), es el hecho de que los huéspedes eligen espontánea y libremente un/a confidente. La confidencia, la empatía y el afecto, permiten encarar la tarea del sentido.

La cuestión del sentido es central para que el/la huésped pueda partir en paz consigo mismo/a. Es necesario decir que, en el ámbito espiritual, además del tema intrapersonal (relación del huésped consigo mismo), está el tema de la relación del huésped con sus allegados y la relación con una eventual trascendencia (religiosa o no).

Respecto de la relación con los demás, es muy importante la cuestión de los vínculos. En el final de la vida, se manifiesta como

\footnotetext{
43 Hay un libro con título similar que recoge esta idea del tiempo final como tiempo precioso, cualificado. Un tiempo que da la oportunidad de realizar cambios positivos para sí y para otros. Ver Jean Monbourquete, El Tiempo Precioso del Final. Aprender a morir (Santander: Sal Terrae, 2005).

44 Entrevista 1, realizada el 16 de Julio de 2014. Las entrevistas que aquí se citan forman parte de la investigación de la beca de posdoctorado que se desarrolla en la Facultad de Teología de la uca, sobre prácticas de espiritualidad popular en el «Hospice San Camilo», como aporte a la reflexión colectiva en el grupo de Investigación sobre «Practicas de Espiritualidad Popular en espacios urbanos (Buenos Aires y Rosario)», perteneciente a la antes dicha Facultad.

Entrevista 3, realizada el 23 de septiembre de 2014.
} 
muy importante el sostén que pueden ofrecer las otras personas, sean familiares, amigos, etc. Esto comienza con la red que es el Hospice: los huéspedes en general están tranquilos y cuidados porque sienten que en la CDE hay una comunidad de sostén. «Estos ángeles están en todo ${ }^{46}$, decía Enrique (un huésped), y se refería a esta red que se ocupaba de los medicamentos, los alimentos, las visitas al hospital, de acompañar a los huéspedes, procurar la cercanía de los familiares, conversar sobre las cuestiones existenciales. Muchas veces, al tener la experiencia de comunidad en la $\mathrm{CDE}$, los huéspedes quieren retomar y reparar relaciones personales heridas o rotas.

Respecto de la trascendencia, los posicionamientos son diversos y ello depende de si se tiene o no una adscripción religiosa o si al menos se asume que la vida continua de alguna forma, más allá de esta existencia. Quienes piensan que todo termina con el último suspiro, igualmente buscan alguna forma de ir más allá, esto suele concretarse con alguna forma de «legado»: el hecho de dejar algo como don o como tarea, es una manera de estar presente en la ausencia, de vincularse con los afectos y de satisfacer la necesidad personal y humana de trascender ${ }^{47}$.

\subsection{El sentido y la narración al final de la vida}

El sentido muchas veces aparece en la narrativa. En el HSC, en las entrevistas hechas a las personas que ofrecen distintos tipos de cuidados aparece mucho la épica del relato ${ }^{48}$, con visos de hagiografía en las historias que cuentan de los huéspedes. Quien narra visualiza

46 Entrevista 8, párrafo 5, realizada el 18 de mayo de 2015.

47 El fundamento de dejar un legado lo describe el Dr. Harvey Chochinov en su libro: Harvey Chochinov, Dignity Therapy. Final Words for Final Days (Oxford University Press: New York, 2012).

48 Cf. Jean Shinoda Bolen, El sentido de la enfermedad (Barcelona: Kairós, 2006). La autora plantea la enfermedad como la posibilidad de iniciar un viaje de indagación y de introspección, en cuyo transcurso la persona se enfrenta con las dificultades de su forma de ser y de su historia. La autora lo muestra como un relato épico, en el que el protagonista es el héroe que tiene que atravesar distintas dificultades. Se muestra también el paralelo con los grandes mitos griegos. Lo interesante es ver de qué manera, cuando la persona se reconoce en su propio relato, y se ve a sí misma atravesando las dificultades, se da cuenta de todo su potencial para pelear también las batallas futuras, se percibe como un héroe o una heroína de su historia personal. Esa conciencia da poder personal y ofrece confianza en la vida. 
para sí y para nosotros como interlocutores, el sentido. De lo sucedido (la historia con sus vericuetos) y de lo hecho (lo que hizo el huésped, lo que hicieron otros o ellos mismos). Para los huéspedes, narrar la historia les ayuda a encontrar el sentido ya sea de su biografía, de partes de su vida o de esta instancia, que se sabe final. Al relatar para otro, muchas veces se cuentan a ellos mismos lo que está sucediendo y van descubriendo fragmentos significativos que les dan señales de que lo vivido vale la pena. También les ayuda a tomar conciencia de sus actitudes positivas, por ejemplo, de su coraje para enfrentar situaciones difíciles, de su fortaleza para resistirlas.

Para voluntarios, voluntarias y personal de salud, relatar les ayuda a tomar conciencia del profundo significado que tiene lo que están haciendo. No porque necesiten justificarse, sino porque al narrar y reencontrar un sentido, hay una conformidad con el camino escogido. Hay un contento íntimo, profundo, algo queda satisfecho por una comprensión existencial. Comprensión que es simultáneamente de la propia vocación y del aporte que se hace a los demás y a Dios. La práctica del voluntariado en el HSC, por ejemplo, tiene sentido para el voluntario/a y a su vez él o ella sienten que eso significa algo importante para los huéspedes.

El sentido de la narrativa, se potencia cuando ella entra en la narrativa que es la Biblia. Cuando nuestras historias se asocian de algún modo a la historia sagrada, nuestras vidas y relatos pequeños se «salvan» al entrar en la historia de la salvación. De hecho, no solo en el Hospice sino en muchos lugares, se escuchan con gusto narraciones bíblicas o de santos: son relatos singulares que se redimen gracias a su ingreso a la gran historia sagrada ${ }^{49}$.

Cuando fallece un huésped, se le pide al/a voluntario/a que ha tenido mayor contacto con la persona que murió, que escriba un pequeño texto y lo envíe por email. Ese texto tiene por finalidad

49 Aquí no podemos desarrollar esta idea, pero véase lo que dice sobre la fuerza salvífica de las historias divinas: Isidor Baumgartner, Psicología Pastoral. Introducción a la praxis de la pastoral curativa (Bilbao: Desclée, 1997), 625-658. 
no solamente comunicar la noticia del fallecimiento, sino recoger algún testimonio sobre el tiempo transcurrido en el Hospice. Cuando se comentó en el HSC que existen estos textos ${ }^{50}$, se dijo que son «despedidas con la Palabra», mencionando que en esos emails se incluía alguna referencia bíblica que por algún motivo se relaciona con la persona. El material pareció sumamente interesante y por ello, en el transcurso de la investigación, se pidió permiso para acceder a algunos de esos textos. Hasta el momento, han facilitado alrededor de 30 A-Dioses, como los llaman, y 2 bienvenidas (las bienvenidas son menos frecuentes, pero a veces se escriben cuando alguien llega a la CDE en especial cuando por algún motivo especial se ha buscado que la persona llegara allí). Sin embargo, no se encontró ninguna cita bíblica, ni directa, ni indirecta. Hay poemas propios de quienes redactan los emails y de autores famosos, pero la Palabra no figura en ninguno de los textos. En el primer momento, se pensó que se trataba de un error de las dos personas que habían referido el tema de la Biblia como integrada a los A-Dioses. Aun tratándose de un error, luego de ir varias veces a la $\mathrm{CDE}$, releer los textos de A-Dioses y de reflexionar sobre el tema, se llegó a una conclusión probable: hay una fuerte percepción de lo sagrado ${ }^{51}$ en el paso de las personas por el Hospice, y que es esa percepción la que hace que los lectores y autores asocien lo escrito con la Palabra de Dios.

En un punto esto es exacto: esos huéspedes han quedado inscriptos en el corazón de los voluntarios con un sesgo no solo afectivo sino espiritual y religioso, por algo titulan a las despedidas «A-Dioses». Evidentemente quien remite cada email ha visto en la historia del/a huésped que despide, una historia de salvación, una historia sagrada, que por la puerta de la Palabra entra (a veces de un modo patente, a veces de un modo misterioso) en la historia de la salvación.

\footnotetext{
50 Esta visita fue compartida con la Prof. María Riquelme quien colaboró en diversas instancias de esta investigación.

51 Dicha percepción de lo sagrado, en el caso de los cristianos (sean voluntarios, profesionales o huéspedes) se asocia con Jesús.
} 
En las narraciones se encuentra el sentido de la historia personal cuando se encuentra el amor. De los padres, de la familia, de Dios. El amor de Dios opera como sintetizador y potenciador, no es infrecuente que se «sospeche» el cuidado amoroso de Dios a través de los cuidados compasivos que ofrecen los voluntarios. Sentirse amado parece que posibilita la sanación de heridas profundas. El desamor y su recuerdo amargo, cuando no han sido trabajados, en cambio, bloquean procesos.

\subsection{Los símbolos y el significado de experiencias que buscan ser sanadas}

En las experiencias religiosas, los símbolos operan como claves de acceso al significado de dichas experiencias. Es muy importante entender el sentido de las prácticas, desentrañando los símbolos. Por ejemplo, en la CDE hay una cantidad de símbolos que son muy propios del lugar y de lo que allí sucede (Las semillas en el frasco de la capilla, la cruz de luces, el legado, los A-Dioses, etc). Aunque cada uno lo vive a su modo, hay significados comunes: las semillas en el frasco significan las oraciones que las personas que pasan por la capilla hacen por los huéspedes, sin embargo mientras que para una de las voluntarias era un elemento decisivo de la experiencia del $\mathrm{HSC}^{52}$, que ella asociaba con la gracia ${ }^{53}$; para otra era «un elemento más» de la capilla. Así vemos que con un significado común, hay significados personales que cualifican o agregan un «plus» a la experiencia.

El ser humano simboliza, es «homo symbolicus» y al simbolizar elabora una experiencia a la vez que la transforma y la redime. ¿̇ué está elaborando, simbolizando, orando y transformando esta persona o grupo a través de esta práctica? Esa práctica y ese símbolo son los que hay que observar, interpretar y acompañar para comprender más a fondo a la persona y secundarla en su camino de sanación. En el

52 Testimonios recogidos el día de la primera celebración de la luz, contando también con la participación de la Prof. María Riquelme, el 17 de diciembre de 2014.

53 De hecho, ella se sentía fortalecida en su tarea por las oraciones de las personas que pasaban por la capilla y ponían esa semilla en el frasco, como símbolo de su oración. 
caso del hospice, una adecuada lectura de los símbolos, potencian las prácticas y las hacen más aptas para acompañar a las personas.

\section{Reflexión conclusiva: la necesidad de repensar las prácticas}

Como apuntara Schneiders y ratificara Liebert, las prácticas parecen no ser consideradas «serias» a la hora de ser incorporadas en el estudio de la espiritualidad en la academia ${ }^{54}$. Quienes utilizan prácticas en sus investigaciones con frecuencia son evaluados como de poco rigor científico y quienes los critican solo le asignan a las prácticas el valor de ejemplos, de relatos meramente subjetivos. Estas valoraciones provienen del desconocimiento, porque de hecho, trabajar adecuadamente con las prácticas requiere contar con una capacitación específica para abordar científicamente las experiencias que se estudian.

Si no se incorporan las prácticas al estudio de la espiritualidad como disciplina académica, las elaboraciones teológicas se alejan de la realidad, no dan cuenta ni relevan la espiritualidad tal y como se desarrolla en la vida de las personas y de las comunidades y, por tanto, se obvia una parte importante de nuestro campo de estudio.

Es necesario incorporarlas en la docencia y en la investigación. Incluir prácticas en clase, genera una inmediata motivación e involucramiento por parte de los alumnos, quienes experimentan de ese modo a la teología como una ciencia capaz de transformar la realidad. Enseña, además, a auto-implicarse en la teología que se está elaborando, enseñanza que se genera en el mismo proceso pedagógico.

En cuanto a la investigación, se requiere afinar la metodología científica para que las herramientas que se utilizan sean adecuadas a la hora de hacer un estudio acreditado académicamente. De hecho, tenemos muchos recursos de investigación teológica para aplicar a 
la hora de leer la Sagrada Escritura, determinados textos históricos, los escritos de los maestros espirituales, y otros materiales. Podemos decir, en cambio, que nuestras mediaciones y recursos científicos para hacer una investigación a partir de las prácticas de espiritualidad, no se encuentran todavía suficientemente desarrollados, en especial si esas experiencias y prácticas son contemporáneas.

La naturaleza interdisciplinar de los mismos conceptos de «práctica»y «experiencia», nos marcan el camino de una metodología intradisciplinar e interdisciplinar para poder trabajar de modo idóneo con estas categorías.

En el campo intradisciplinar, el primer diálogo que se presenta a raíz del estudio sobre «prácticas y experiencias», es el de la teología pastoral. Un trabajo conjunto sobre diversas prácticas, produce un acercamiento teológico mucho más completo, que no solo permite avanzar sobre la investigación concreta que se lleva adelante, sino sobre la misma naturaleza del diálogo intradisciplinar ${ }^{55}$.

En el campo interdisciplinar, las ciencias sociales son las grandes interlocutoras de las experiencias y de las prácticas espirituales. Metodológicamente la investigación cualitativa, que se utiliza mucho en dichas ciencias, es un instrumento muy valioso a la hora del relevamiento y del análisis de nuestros materiales de estudio ${ }^{56}$. En el presente estudio de caso, el método cualitativo y las herramientas asociadas al mismo, permitieron llegar más lejos y de un modo más profundo en la rica experiencia del HSC.

La espiritualidad a nivel académico no puede desarrollarse sin el estudio de las prácticas ya que, ese estudio conduce a fecundos diálogos intra e interdisciplinares. No solo la teología académica se va a enriquecer con el estudio de las prácticas, sino las mismas prácticas y, obviamente, quienes las llevan adelante, se verán enriquecidos y

55 Un ejemplo de esto es el acercamiento al tema que hace Claire Wolfteich, «Animating Questions: Spirituality and Practical Theology», 121-143.

56 Cf. Virginia Azcuy, coord. Ciudad Vivida. Prácticas de Espiritualidad en Buenos Aires (Buenos Aires: Guadalupe, 2014). 
dinamizados ${ }^{57}$, desarrollando otra vía para la renovación pastoral que nos propone el Papa Francisco.

\section{Bibliografía}

Azcuy, Virginia, Coord. Ciudad Vivida. Prácticas de Espiritualidad en Buenos Aires. Buenos Aires: Guadalupe, 2014.

Azcuy, Virginia. «La Espiritualidad como Disciplina Teológica. Panorama Histórico, Consensos y Perspectivas actuales». Teología 105 (2011): 251-280.

Baumgartner, Isidro. Psicología Pastoral. Introducción a la praxis de la pastoral curativa. Bilbao: Desclée, 1997.

Bellet, Maurice. Un trajet vers l'essentiel. Paris: Seuil, 2004.

Benito, Enric; Barbero, Javier; Dones, Mónica y Gómez, Julio. Espiritualidad en Clínica. Una propuesta de Evaluación y Acompañamiento Espiritual en Cuidados Paliativos. Buenos Aires: Ed Biblos - Instituto Pallium Latinoamérica, 2015.

Childs, Brian. «Experience». En Dictionary of Pastoral Care and Counseling, editado por Rodney J. Hunter, 388. Nashville: Abingdon Press, 1990.

Chochinov, Harvey. Dignity Therapy. Final Words for Final Days. Oxford University Press: New York, 2012.

Chopp, Rebecca. Saving Work: Feminist Practices in Theological Education, Louisville Westminster: John Knox, 1995.

Cursach Salas, Rosa. «Experiencia. La experiencia de las mujeres en la teología feminista. Prácticas de teología fundamental feminista». En 10 palabras clave en Teología Feminista, editado por Mercedes Navarro y Pilar de Miguel, 17-55. Navarra: Verbo Divino, 2004.

57 Así lo señalaron los voluntarios de HSC en la reunión del 30 de agosto de 2016, al exponerles algunos resultados de la investigación que estoy desarrollando en el Hospice. 
Dyckman, Katherine; Garvin, Mary y Liebert, Elizabeth. The Spiritual Exercises Reclaimed: Uncovering Liberating Possibilities for Women. New York: Paulist Press, 2001.

Frohlich, Mary. «Spiritual Discipline, Discipline of Spirituality: Revisiting Questions of Definition and Method». Spiritus 1, Vol. 1 (2001): 65-78.

Ganzevoort, Ruard y Roeland, Johan. «Lived religion: the praxis of Practical Theology». IJPT 18 Vol. 1 (2014): 91-101.

Goffi, Tullo. La experiencia espiritual, hoy. Líneas esenciales de la espiritualidad cristiana contemporánea. Salamanca: Sígueme, 1987.

Holder, Arthur y Dahill, Lisa. «Teaching Christian spirituality in seminaries today». Christian Spirituality Bulletin 7-2 (1999): 9-12.

Hospice San Camilo. Consultada en diciembre 30, 2016. http://www. hospicesancamilo.org.ar.

Lescher, Bruce y Liebert, Elizabeth. Exploring Christian Spirituality: Essays in Honor of Sandra Schneiders. New York: Paulist Press, 2006.

Liebert, Elizabeth. Changing Life Patterns: Adult Development in Spiritual Direction. New York: Paulist Press, 1992; Revised Edition, Atlanta: Chalice Press, 2000.

Liebert, Elizabeth. «Practice». En The Blackwell Companion in Christian Spirituality, editado por Arthur Holder, 496-514. Chichester, West Sussex: Blackwell Publishing, 2005.

Liebert, Elizabeth. «The Role of Practice in the Study of Christian Spirituality». En Minding the Spirit. Editado por Elizabeth Dreyer y Mark Burrows, 79-99. Baltimore: John Hopkins University Press, 2005. 
Liebert, Elizabeth. The Soul of discernment. A Spiritual Practice for Communities and Institutions. Louisville, Kentucky: Westminster John Knox Press, 2016.

Liebert, Elizabeth. The Way of Discernment. Spiritual Practices for Decision Making. Louisville, Kentucky: Westminster John Knox Press, 2008.

Liebert, Elizabeth y Endres, John. A Retreat with the Psalms: Resources for Personal and Communal Prayer. New York: Paulist Press, 2001.

Mazzini, María Marcela. «El Cuidado Hospice como Acompañamiento espiritual. Estudio de caso: "Hospice San Camilo"», Teología 122, Vol. LIV (2017): 69-97.

Monbourquete, Jean. El Tiempo Precioso del Final. Aprender a morir. Santander: Sal Terrae, 2005.

Pikaza, Xavier. Experiencia religiosa y cristianismo. Introducción al misterio de Dios. Salamanca: Sígueme, 1981.

Puchalski, Christina. «La mejora de la calidad de los cuidados espirituales como una dimensión de los cuidados paliativos: el informe de la Conferencia de Consenso». Medicina Paliativa 18, Vol.1 (2011): 55-78.

Rodríguez Panizo, Pedro. «Experiencia religiosa». En Nuevo Diccionario de Teología, dir. Juan José Tamayo, 358-367. Madrid: Trotta, 2005.

Schneiders, Sandra. «A Hermeneutical Approach to the Study of Christian Spirituality». En Minding the Spirit, editado por Elizabeth Dreyer - Mark Burrows, 49-60. Baltimore: John Hopkins University Press, 2005.

Schneiders, Sandra. «Spirituality in the Academy». Theological Studies 50, (1989): 676-697.

Shinoda Bolen, Jean. El sentido de la enfermedad. Barcelona: Kairós, 2006. 
Volf, Miroslav y Bass, Dorothy. Practicing Theology: Beliefs and Practices in Christian Life. Michigan-Cambridge: Eerdmans Publishing, 2002.

Von Balthasar, Hans Urs. Gloria. Una estética teológica. Madrid: Encuentro, 1985.

Waaijman, Kees. «Toward a phenomenological definition of Spirituality». Studies in Spirituality 3, (1993): 5-56.

Wolfteich, Claire. «Animating Questions: Spirituality and Practical Theology». IJPT 13, (2009): 121-143.

Enviado: 3 de enero de 2017 Aceptado: 2 de marzo de 2017 